\title{
Construção e Análise das Redes Sociais de Personagens dos Filmes da Franquia $O$ Senhor dos Anéis
}

\author{
Victoria Patrícia Aires, Paulo Rodrigo Martins, Fabiola Nakamura \\ Instituto de Computação - Universidade Federal do Amazonas (UFAM) \\ 69077-000 - Manaus - AM - Brasil \\ \{vpsa,prom, fabiola\}@icomp.ufam.edu.br
}

\begin{abstract}
This article focuses on character networks, which are social networks that presents complex network properties. The strategy to build the network of characters of a movie is using the same script, that contains information such as the beginning of a scene, the characters that appear and dialogues. Usually, algorithms to build the social network considers only characters who have speeches in scene. In this paper, we present a metodology that also considers the characters that were quoted or just appear in the scene. We use the Lord of the Rings franchise to apply the metodology, building and analysing the character networks, answering who are the protagonists according to three centrality measures: weighted degree, betweeness and PageRank.
\end{abstract}

Resumo. Este artigo tem como foco as redes de personagens, que são um tipo de rede social, apresentando propriedades de redes complexas. Para construir a rede de personagens de um filme, uma estratégia é usar o script do mesmo, que contém informações como o início de uma cena, os personagens que aparecem e diálogos. Em geral, a construção da rede social leva em conta apenas os personagens que possuem falas em cada cena. Neste trabalho, apresentamos uma metodologia que leva em consideração também personagens citados ou que apenas aparecem nas cenas. Utilizamos os filmes da franquia $O$ Senhor dos Anéis para a aplicação da metodologia, construindo a rede de personagens e posteriormente analisando, respondendo quem são os protagonistas de acordo com três medidas de centralidade: grau ponderado, intermediação e PageRank.

\section{Introdução}

Redes sociais são redes que modelam relacionamentos e interações sociais, tais como amizades, trabalho e colaboração em artigos. Neste contexto, existem as redes de personagens, um tipo particular de rede social. Elas representam os relacionamentos entre personagens de um filme. Sendo um tipo de rede social, elas possuem características de redes complexas, como atributos de mundo pequeno (small world), comunidades bem definidas e distribuição desigual de grau [Bonato et al. 2016]. Além disso, possuem a particularidade de refletir o enredo do filme, ao tornar explícitas as conexões entre personagens, destacando núcleos formados ao longo da história através de suas comunidades. Estas redes encontram correlações com situações no mundo real, como a representação de uma rede de criminosos, uma rede de políticos, ou outras situações onde existem relacionamentos documentados que possuem dinamicidade e evolução temporal [Spadon et al. 2016].

$\mathrm{Na}$ literatura, existem trabalhos que extraem redes sociais de personagens de obras literárias e cinematográficas. No caso específico dos filmes, a extração é feita a partir 
do script do filme. O site Movie Galaxies [Kaminski et al. 2012] contém redes sociais extraídas de diversos filmes. Porém, a extração é feita observando apenas os personagens que falam em cada cena. Assim, personagens importantes, mas que não possuem falas, não são representados na rede social e o resultado final pode não ser uma modelagem adequada.

Neste trabalho, o objetivo é propor uma metodologia para construção de redes de personagens de filmes, dados o script e a lista de personagens. Esta construção leva em conta os personagens que dialogam nas cenas ou apareceram, porém sem falar. Desta forma, é possível construir uma rede de personagens mais abrangente, incluindo personagens sem falas (como animais e robôs, por exemplo). Aplicamos a estratégia aos três filmes da franquia $O$ Senhor dos Anéis e também à trilogia como um todo, e após análise, responde-se quem são os protagonistas do filme de acordo com as medidas de centralidade (grau, intermediação e PageRank). A franquia O Senhor dos Anéis foi escolhida devido à estrutura de seu enredo, uma jornada onde as interações e influência dos personagens mudam através dos filmes. Acreditamos que, devido a esta dinamicidade, é interessante analisar de que forma os filmes são modelados em redes complexas e verificar o quanto estas redes refletem o enredo dos filmes.

As principais contribuições deste trabalho são: apresentação de uma metodologia para extração da rede social de personagens de filmes, utilizando o script e a lista de personagens do mesmo. Diferente de outros trabalhos presentes na literatura, esta metodologia leva em consideração tanto personagens que possuem falas quanto os que são apenas citados ou aparecem em uma cena. Ao final, a metodologia é aplicada aos três filmes da franquia O Senhor dos Anéis para construção das redes de personagens e posterior análise por meio de medidas de centralidade em redes complexas, revelando quem são os personagens protagonistas de cada filme e da trilogia como um todo.

O restante do artigo está organizado da seguinte forma: a Seção 2 apresenta uma visão geral dos trabalhos relacionados. A Seção 3 aponta como foi feita a modelagem das redes de personagens. A Seção 4 traz as métricas utilizadas durante o trabalho. Já a Seção 5 contém os resultados obtidos e, por fim, a Seção 6 traz as considerações finais, seguida das referências.

\section{Trabalhos Relacionados}

Esta seção sumariza alguns trabalhos que realizam extração de redes de personagens de filmes. Eles se diferenciam principalmente pela técnica utilizada, envolvendo conceitos de aprendizagem de máquina, reconhecimento facial e expressões regulares.

Em [Park et al. 2012] o foco está em construir a rede de personagens levando em conta aqueles que falam em cada cena e classificá-los quanto a papéis principais, secundários e extras. É realizada a sincronização entre o script do filme e as legendas. $\mathrm{O}$ lado positivo desta abordagem está no uso da centralidade de grau (degree centrality) para classificar os personagens em papéis. O lado negativo é que alguns foram incorretamente classificados, até mesmo personagens importantes, pois o contexto dos diálogos e ações não é levado em conta, o que acabou causando uma falha no método.

O trabalho de [Weng et al. 2007] também é baseado em papéis (roles). A rede social de papéis é usada para identificar comunidades e detectar suas relações com o enredo. 
São usadas técnicas de reconhecimento facial para determinar os nós (roles) da rede. O cenário escolhido compreende apenas filmes bilaterais, geralmente com comunidades que representam o bem e o mal. São identificados nós determinantes (leading roles) na rede de papéis através de medidas de centralidade de grau. Então, determina-se as comunidades que circundam cada uma das leading roles. O artigo possui certo viés, uma vez que analisa apenas filmes bilaterais, com uma estrutura praticamente pré-determinada. Por exemplo, filmes que contam com o lado do bem e do mal bem definidos, onde o caráter dos personagens é explícito. Além disso, a detecção de cenas e reconhecimento facial foram feitas manualmente, sendo assim inapropriadas ao presente contexto.

Já em [Agarwal et al. 2014], a estratégia é receber um script e assinalar à cada linha uma tag indicando se a mesma é o início de uma cena, uma descrição de cena, o nome de um personagem, um diálogo ou um meta-dado sobre o script. Isto é utilizado para a construção da rede social de personagens de um filme. A abordagem escolhida é aplicar uma técnica de aprendizagem de máquina para realizar esta marcação no script, comparando com o método de expressões regulares. A rede foi construída ao adicionar um link a dois personagens que aparecem em uma mesma cena. Esta técnica não leva em conta personagens que foram apenas citados ou não possuem falas, o que pode levar a uma representação incorreta da importância de um personagem. Outro problema está no fato de personagens aparecerem no script com apelidos ou pseudônimos. Estes pontos serão tratados no presente trabalho.

\section{Modelagem da Rede}

Uma rede social é uma espécie de rede complexa. Estas redes possuem as seguintes características [Shen 2013]:

- A distribuição de grau dos vértices é desigual e segue a lei de potência: há muitos vértices de grau pequeno, e poucos vértices com muitas conexões. Isto faz com que a importância (ou centralidade) dos vértices varie na rede.

- Existe a propriedade small-world (mundo pequeno): a distância entre quaisquer dois vértices na rede é pequena em relação ao número total de vértices e arestas total.

- Há estrutura de comunidades bem definida: alguns elementos são mais conectados entre si do que com outros na rede, formando agrupamentos ou clusters.

No contexto de redes sociais, que modelam interações sociais entre um grupo de indivíduos, estas características podem indicar quais os indivíduos mais influentes na rede; o quanto duas pessoas que não se conhecem estão próximas; e quais os indivíduos associados, direta ou indiretamente, por meio de interesses em comum ou similaridades entre si.

A extração da rede social de cada filme tem como base o script ou screenplay do mesmo. Um script contém a descrição de cada cena, bem como os personagens que aparecem e as falas daqueles que se manifestam nela. Estas informações estão dispostas seguindo um formato semi-regular, permitindo o uso de um programa baseado em expressões regulares para extrair os dados necessários para a construção da rede social. A Figura 1 apresenta um exemplo de uma cena em um script.

Neste trabalho, fazemos uso do script e da lista de personagens do filme para compor a rede social de personagens. Para a execução dos experimentos, foram utiliza- 


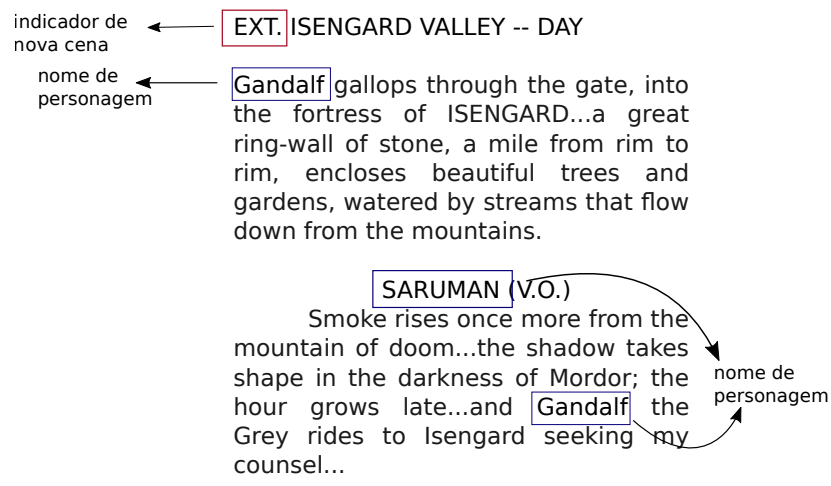

Figura 1. Trecho de um script do filme A Sociedade do Anel. Em destaque, a marcação EXT, indicadora de início de cena, além dos personagens que falam ou estão sendo mencionados.

dos scripts da franquia O Senhor dos Anéis, obtidos do site IMSDb [IMSDb 2017], que disponibiliza os roteiros de um grande número de filmes. Neste contexto, foi utilizada a lista de personagens de cada filme da franquia, obtida do site IMDb [IMDb 2017], que cataloga grande parte da produção cinematográfica mundial. Em resumo, a lógica é procurar uma nova cena, e acrescentar arestas entre os personagens que aparecem (ou foram citados) nela. Caso já exista aresta, o peso da mesma é incrementado.

Algoritmo 1: Parser para extração da rede de personagens de filmes

Entrada: Script e lista de personagens do filme.

Saída: Rede social de personagens.

Dados: cenaAtual

início

cenaAtual $\leftarrow \emptyset$;

para cada linha do script faça

se linha começa com $I N T \mid E X T$ então

//se existe uma cena anterior, adicionar relações

para cada personagem $p$ em cenaAtual faça

para cada personagem $q$ em cenaAtual faça

se $p \neq q$ então

adiciona aresta entre $p$ e $q$ (ou incrementa o peso da aresta

existente);

fim se

fim para cada

fim para cada

//prepara o conjunto para nova cena

cenaAtual $\leftarrow \emptyset$;

fim se

senão

para cada personagem na lista de personagens faça

se personagem aparece na linha \& personagem $\notin$ cenaAtual então

$\mid$ cenaAtual $\leftarrow$ cenaAtual $+\{$ personage $\}$;

fim se

fim para cada

fim se

fim para cada

fim 


\section{Medidas de Centralidade}

Para analisar as redes sociais de cada filme como redes complexas, são aplicadas medidas de centralidade. Estas respondem quem são os nós principais ou mais influentes da rede, cada uma seguindo um critério específico. As medidas escolhidas para análise neste trabalho são [Perra and Fortunato 2008]:

- Centralidade de grau ponderado (weighted degree centrality): considera que os nós de maior grau são mais centrais. O grau de um vértice é a soma dos pesos das arestas a que ele está conectado. No contexto deste trabalho, considera que os personagens mais importantes são os que possuem mais interações com os demais.

- Centralidade de intermediação (betweenness centrality): indica o número de vezes que um vértice está no caminho mínimo entre dois outros vértices quaisquer na rede. Isso significa que a importância aumenta conforme ele funciona como ponte para conectar outros vértices na rede. Assim, um personagem é mais central quando conecta personagens de diversos núcleos diferentes da história.

- Centralidade de PageRank (PageRank centrality): considera o número e a qualidade de conexões de um vértice para determinar a importância do mesmo. Vértices mais importantes são os que recebem mais conexões de outros vértices importantes. No caso dos filmes, os personagens centrais interagem com personagens importantes na história.

\section{Resultados Obtidos}

Para realizar os experimentos da estratégia proposta, foram feitos testes utilizando o software Gephi [Gephi 2017], que calcula as medidas de centralidade e permite a modelagem visual dos grafos que representam as redes e implementação em Python 3 para o parser do script. As redes de personagens construídas foram as dos três filmes da franquia $O$ Senhor dos Anéis: A Sociedade do Anel, As Duas Torres e O Retorno do Rei. Além disso, foi construída uma quarta rede de personagens, representando os três filmes. A franquia $\mathrm{O}$ Senhor dos Anéis foi escolhida devido à dinamicidade do enredo: os personagens iniciam uma caminhada juntos no primeiro filme, separam-se em diferentes enredos no segundo e, por fim, juntam-se no último filme. Também é interessante o fato dos personagens adquirirem importâncias diferentes no decorrer dos três filmes, devido aos enredos em que se envolvem.

A história de Senhor dos Anéis conta a saga de vários habitantes de um mundo fictício denominado Terra Média, que está sob ameaça de um poderoso mago chamado Sauron, o Senhor dos Anéis. Sauron criou um anel muito poderoso, o Anel do Poder, capaz de prover a seu mestre o domínio completo sobre os outros seres deste mundo. Porém, Sauron não detém o anel, que está em posse de Frodo, um hobbit (um ser semelhante aos humanos, porém de estatura muito menor).

Os resultados levam em conta apelidos e pseudônimos que aparecem durante a história: por exemplo, o personagem Aragorn é chamado, durante parte dos filmes, de Passolargo. Samwise, Meriadoc e Peregrin são os nomes próprios dos personagens Sam, Merry e Pippin, que são chamados pelos apelidos durante a maior parte dos filmes. Há um caso especial: o personagem Gollum era chamado no passado de Smeagol, e o personagem sofre um distúrbio de personalidade, em uma luta interna entre Gollum e Smeagol. Pelo fato de um dialogar com o outro, mantivemos os vértices Gollum e Smeagol como 
distintos. Além disso, o fato do vilão Sauron não possuir uma forma corpórea e, deste modo, não possuir falas, não o impede de ser representado na rede social, pois ele é citado por outros personagens. Esta é uma fraqueza encontrada em outros trabalhos, que incluem apenas personagens com fala explícita e não levam em consideração apelidos e nomes próprios, criando vértices excedentes.

\subsection{A Sociedade do Anel}

No primeiro filme da saga, Frodo inicia sua caminhada em direção a Mordor, o lugar onde o Anel do Poder de Sauron foi feito e o único onde pode ser destruído. Em sua companhia, vão seus melhores amigos, Sam, Merry e Pippin (todos hobbits), além de Gandalf (um mago), Legolas (um elfo), Gimli (um anão), Aragorn (chamado também de Passolargo, um homem) e Boromir (também homem). Juntos, estes personagens formam a Sociedade do Anel. Observa-se nas redes construídas que a interação entre os personagens da Sociedade do Anel é de fato muito forte.

Há três comunidades identificadas, representadas através das cores dos vértices na Figura 2. Cada uma representa uma parte do enredo do filme, com destaque para a comunidade em laranja, contendo quase toda a Sociedade do Anel e outros hobbits. A comunidade em roxo inclui personagens do núcleo de Valfenda e Lórien (elfos), o vilão Sauron e outros relacionados à história do anel, como a criatura Gollum, que possuiu o anel por muitos anos. Já a comunidade em verde destaca os membros da sociedade do anel mais envolvidos em batalha: Boromir, Legolas e Gimli, que lutaram contra os orcs Uruk-Hai, liderados pelo mago Saruman e o orc Lurtz. As medidas de centralidade foram utilizadas para classificar os personagens do modo apresentado na Tabela 1.

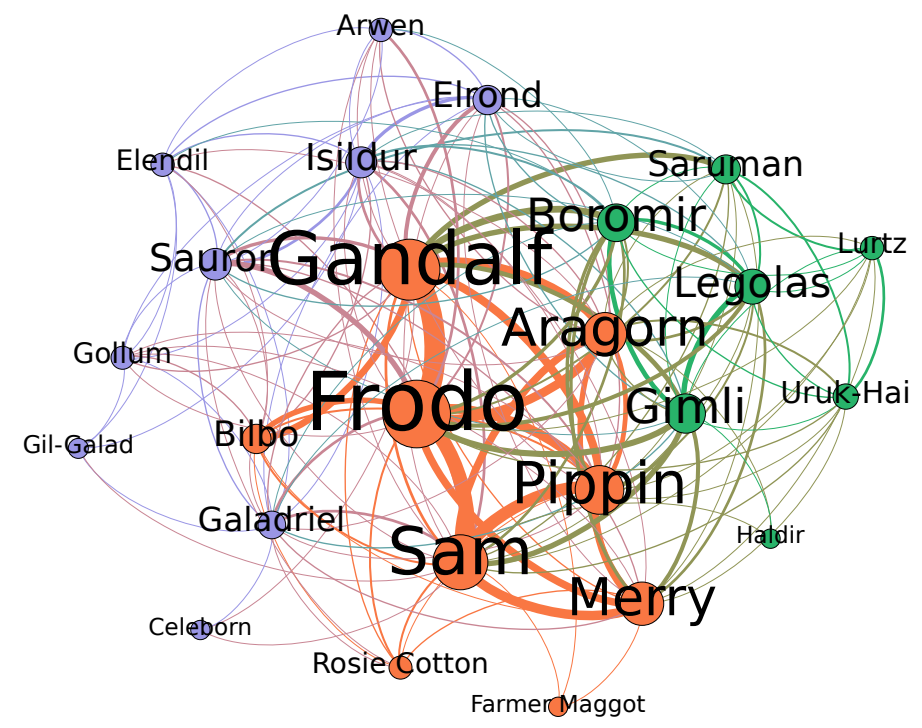

Figura 2. Rede de personagens de A Sociedade do Anel classificados de acordo com a centralidade de grau ponderado.

Analisando os resultados da Tabela 1, verificamos que segundo o grau ponderado e PageRank, os personagens Frodo, Gandalf e Sam são os principais destaques. Isto significa que estes personagens possuem alto número de conexões. Além disto, estes personagens estão conectados com outros de grande importância. Isto faz sentido, pois são os três personagens principais da Sociedade do Anel, interagindo com outros personagens 
Tabela 1. Classificação de personagens segundo as medidas de centralidade aplicadas à rede social do filme A Sociedade do Anel.

\begin{tabular}{|l|l|}
\hline Personagem & $\begin{array}{l}\text { Grau } \\
\text { ponderado }\end{array}$ \\
\hline Frodo & 148.0 \\
\hline Gandalf & 128.0 \\
\hline Sam & 110.0 \\
\hline Pippin & 91.0 \\
\hline Merry & 75.0 \\
\hline Aragorn & 75.0 \\
\hline Gimli & 63.0 \\
\hline Boromir & 58.0 \\
\hline Legolas & 50.0 \\
\hline Sauron & 40.0 \\
\hline
\end{tabular}

\begin{tabular}{|l|l|}
\hline Personagem & Betweenness \\
\hline Sam & 0.15485 \\
\hline Gandalf & 0.05249 \\
\hline Galadriel & 0.04168 \\
\hline Merry & 0.03906 \\
\hline Aragorn & 0.03271 \\
\hline Isildur & 0.02399 \\
\hline Pippin & 0.02365 \\
\hline Frodo & 0.02365 \\
\hline Gimli & 0.02012 \\
\hline Sauron & 0.01667 \\
\hline
\end{tabular}

\begin{tabular}{|l|l|}
\hline Personagem & PageRank \\
\hline Frodo & 0.11629 \\
\hline Gandalf & 0.10391 \\
\hline Sam & 0.09349 \\
\hline Pippin & 0.07268 \\
\hline Merry & 0.06273 \\
\hline Aragorn & 0.06272 \\
\hline Gimli & 0.05347 \\
\hline Boromir & 0.04917 \\
\hline Legolas & 0.04274 \\
\hline Sauron & 0.03696 \\
\hline
\end{tabular}

importantes do mesmo núcleo. Outra observação interessante é o fato de Sam possuir o maior betweenness: isto significa que ele é um personagem intermediador de núcleos. Podemos perceber isto na história ao verificar que Sam é a ponte de conexão entre os demais hobbits comuns e o núcleo de Frodo. Gandalf e Galadriel também conectam núcleos diferentes: o dos hobbits com o de Valfenda e Lórien, respectivamente, núcleos de elfos. Assim, podemos considerar como protagonistas Frodo, Gandalf e Sam.

\subsection{As Duas Torres}

No segundo filme, Frodo e Sam decidem prosseguir sozinhos, encontrando depois Gollum, um ser interessado no anel. Enquanto isso, Merry e Pippin são sequestrados. Legolas, Gimli e Aragorn vão para o reino de Rohan. Lá, conhecem Theoden (o rei) e Eowyn (sobrinha do rei). Neste filme, o mago Saruman, aliado de Sauron, inicia um ataque a Rohan, enviando seu exército de Uruk-Hai (orcs). O rei Theoden defende o reino contando com a ajuda de Aragorn, Gandalf, Gimli e Legolas.

A rede social do filme, representada na Figura 3, possui três comunidades bem definidas e relacionadas ao roteiro. A comunidade em laranja mostra o núcleo de Frodo, Sam e Gollum em sua caminhada para Mordor. Em roxo, encontram-se os personagens em Rohan. Em verde, o núcleo de Merry e Pippin, que estavam próximos ao antagonista Saruman.

Tabela 2. Classificação de personagens segundo as medidas de centralidade aplicadas à rede social do filme As Duas Torres.

\begin{tabular}{|l|l|}
\hline Personagens & $\begin{array}{l}\text { Grau } \\
\text { ponderado }\end{array}$ \\
\hline Aragorn & 112.0 \\
\hline Sam & 81.0 \\
\hline Theoden & 77.0 \\
\hline Legolas & 66.0 \\
\hline Frodo & 64.0 \\
\hline Gimli & 62.0 \\
\hline Saruman & 59.0 \\
\hline Uruk-Hai & 56.0 \\
\hline Gandalf & 55.0 \\
\hline Gollum & 40.0 \\
\hline
\end{tabular}

\begin{tabular}{|l|l|}
\hline Personagens & Betweenness \\
\hline Saruman & 0.14261 \\
\hline Aragorn & 0.11561 \\
\hline Uruk-Hai & 0.09188 \\
\hline Sauron & 0.07877 \\
\hline Sam & 0.05352 \\
\hline Gandalf & 0.04741 \\
\hline Gimli & 0.03791 \\
\hline Theoden & 0.03789 \\
\hline Faramir & 0.02659 \\
\hline Legolas & 0.01770 \\
\hline
\end{tabular}

\begin{tabular}{|l|l|}
\hline Personagens & PageRank \\
\hline Aragorn & 0.08583 \\
\hline Sam & 0.06013 \\
\hline Theoden & 0.05938 \\
\hline Saruman & 0.05065 \\
\hline Legolas & 0.04994 \\
\hline Gimli & 0.04803 \\
\hline Uruk-Hai & 0.04778 \\
\hline Frodo & 0.04759 \\
\hline Gandalf & 0.04316 \\
\hline Sauron & 0.03304 \\
\hline
\end{tabular}




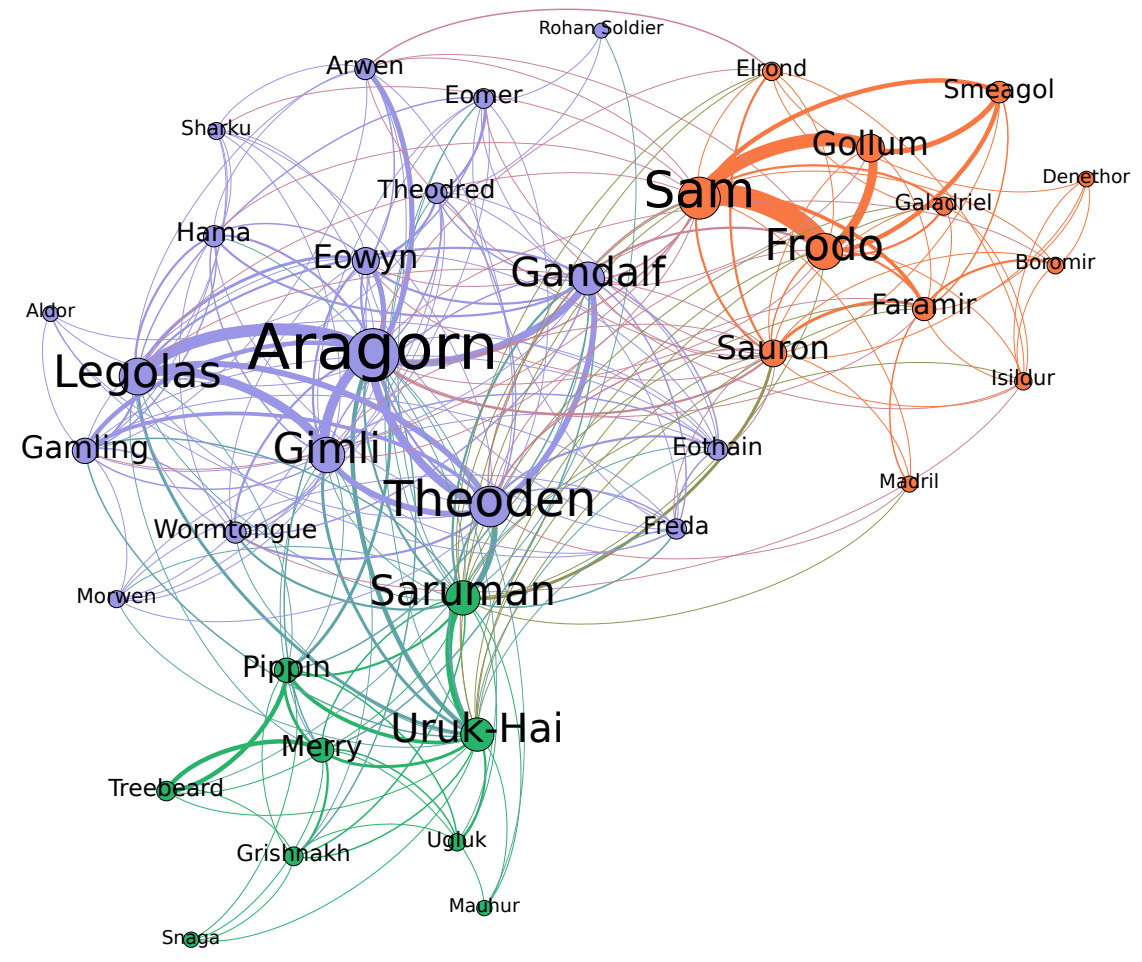

Figura 3. Rede de personagens de As Duas Torres classificados de acordo com a centralidade de grau ponderado.

A classificação de acordo com as medidas de centralidade, presente na Tabela 2, mostra que Aragorn, Sam e Theoden são os personagens com maiores valores de centralidade segundo grau ponderado e PageRank. Neste filme, os núcleos de maior destaque são os da batalha do reino de Rohan e o de Frodo e Sam caminhando para Mordor. Desta forma, faz sentido que os personagens principais façam parte destes núcleos, onde estão presentes outros personagens importantes como Legolas e Gimli. Um fato interessante é Sam obter destaque maior que Frodo. Embora estejam caminhando juntos, neste filme há um número maior de cenas envolvendo apenas Sam e Gollum, justificando a interação mais intensa entre os dois personagens capturada pela rede social.

A pontuação de betweenness indica que Saruman é o personagem intermediador. Mais uma vez, isto é equivalente ao roteiro, pois este personagem age em favor de Sauron, sendo responsável pela batalha em Rohan. Assim, ele conecta núcleos importantes: o de Isengard e o de Rohan. Pelo mesmo motivo, os Uruk-Hai (exército de Sauron) aparecem em terceiro. Aragorn em segundo lugar indica seu papel de intermediador entre os personagens da realeza de Rohan, os remanescentes da Sociedade do Anel e os de Valfenda. Por fim, podemos considerar como protagonistas Aragorn, Sam e Theoden.

\subsection{O Retorno do Rei}

No último filme acontece a batalha pelo anel e pela proteção do reino de Gondor, do qual Aragorn se mostra herdeiro ao trono. Merry e Pippin participam de núcleos diferentes. Aragorn, Legolas, Gimli e Gandalf possuem papel importante na guerra. Enquanto isso, Frodo, Sam e Gollum chegam a Mordor, onde o anel é finalmente destruído, para a derrota de Sauron. 


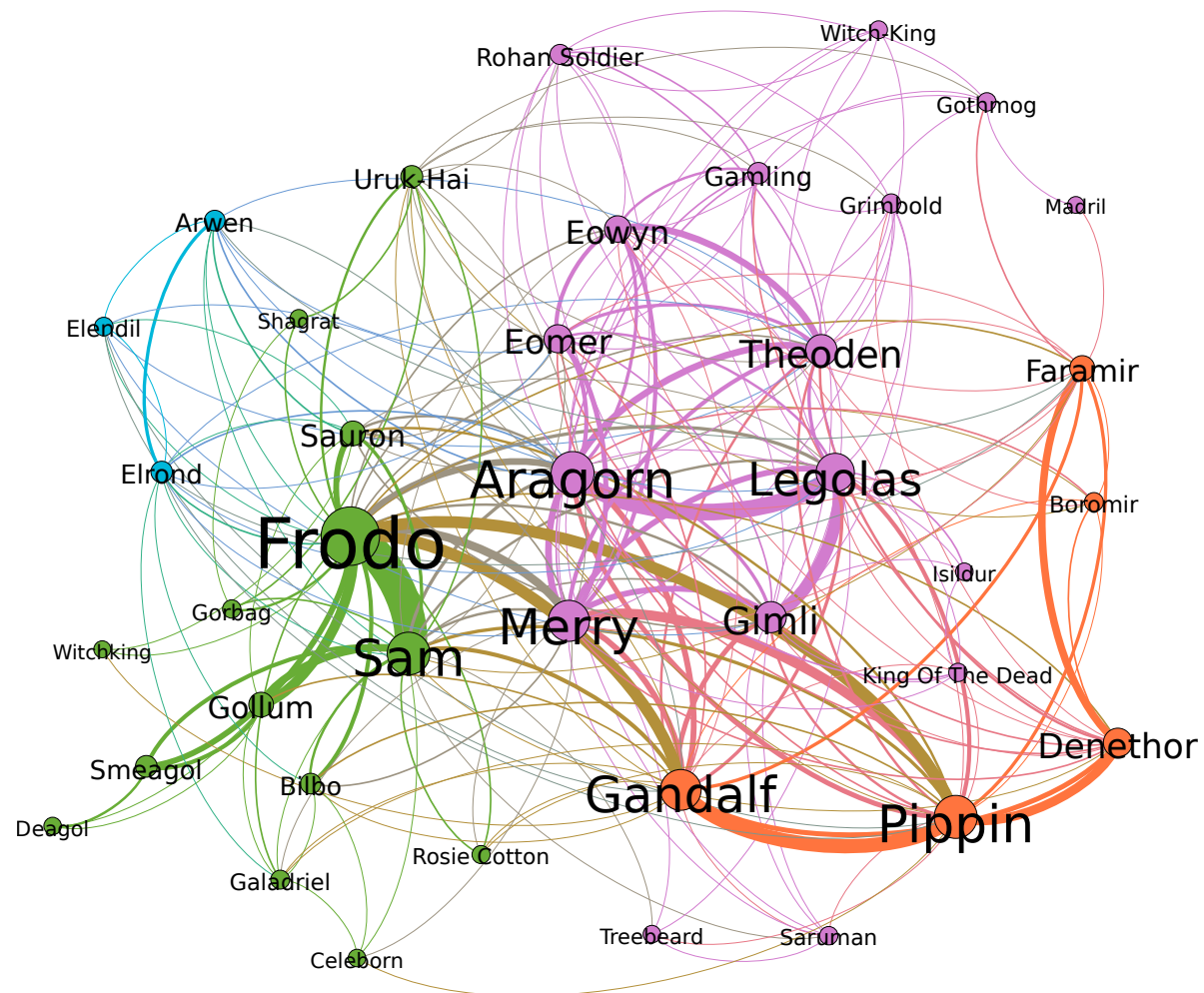

Figura 4. Rede de personagens de O Retorno do Rei classificados de acordo com a centralidade de grau ponderado.

A Figura 4 apresenta a rede social do filme, com os vértices classificados de acordo com a centralidade de grau. Desta vez, foram identificadas quatro comunidades: em azul, os personagens do núcleo dos elfos em Valfenda. Em verde, os personagens relacionados à Mordor e o anel. Em laranja, o núcleo do regente de Gondor, Denethor, envolvendo Gandalf e Pippin. Em roxo, os personagens que lutaram na batalha de Gondor, com destaque para Aragorn, Merry, Legolas e Gimli.

Tabela 3. Classificação de personagens segundo as medidas de centralidade aplicadas à rede social do filme $O$ Retorno do Rei.

\begin{tabular}{|l|l|}
\hline Personagens & $\begin{array}{l}\text { Grau } \\
\text { ponderado }\end{array}$ \\
\hline Frodo & 153.0 \\
\hline Aragorn & 97.0 \\
\hline Pippin & 97.0 \\
\hline Sam & 96.0 \\
\hline Merry & 90.0 \\
\hline Gandalf & 89.0 \\
\hline Legolas & 80.0 \\
\hline Theoden & 55.0 \\
\hline Gimli & 54.0 \\
\hline Denethor & 46.0 \\
\hline
\end{tabular}

\begin{tabular}{|l|l|}
\hline Personagens & Betweenness \\
\hline Frodo & 0.18196 \\
\hline Merry & 0.08323 \\
\hline Sam & 0.07273 \\
\hline Gandalf & 0.06315 \\
\hline Aragorn & 0.05734 \\
\hline Faramir & 0.05321 \\
\hline Pippin & 0.05310 \\
\hline Legolas & 0.05137 \\
\hline Uruk-Hai & 0.03941 \\
\hline Theoden & 0.03511 \\
\hline
\end{tabular}

\begin{tabular}{|l|l|}
\hline Personagens & PageRank \\
\hline Frodo & 0.10697 \\
\hline Aragorn & 0.06769 \\
\hline Sam & 0.06721 \\
\hline Pippin & 0.06680 \\
\hline Merry & 0.06325 \\
\hline Gandalf & 0.06149 \\
\hline Legolas & 0.05638 \\
\hline Theoden & 0.04088 \\
\hline Gimli & 0.03877 \\
\hline Denethor & 0.03360 \\
\hline
\end{tabular}

A Tabela 3 mostra a classificação dos personagens de acordo com as medidas de centralidade. Observa-se que Frodo foi o personagem que obteve maior pontuação nas três. Este é um fato interessante, pois diferente dos outros dois filmes, Frodo é o perso- 
nagem com maior número de conexões (e com mais qualidade) e o intermediador. Há uma correlação com o enredo, pois neste filme, Frodo é altamente citado pelos personagens envolvidos na batalha de Gondor. Assim, ele conecta os núcleos de Mordor e Rohan/Gondor, e simultaneamente, adquire muitas conexões.

Aragorn teve alta classificação em duas métricas, assim como Sam. Aragorn tem papel de liderança na batalha de Gondor, interagindo com diversos personagens. Sam, por sua vez, é fundamental no núcleo de Mordor, auxiliando Frodo a destruir o Anel. Desta forma, as medidas indicam que Frodo, Aragorn e Sam são os protagonistas da história. É interessante observar que outros personagens como Pippin e Merry também obtiveram pontuações parecidas, o que corresponde à participação dos mesmos no filme. Cada um destes personagens possui um papel chave em seus determinados pontos da história.

\subsection{A Trilogia Completa}

A rede social que reúne os três filmes é apresentada na Figura 5. Esta rede foi construída ao unir em uma única rede as obtidas de cada filme individualmente. Nesta rede social, percebemos três comunidades. A comunidade em roxo é composta pelos personagens do núcleo das guerras de Rohan e Gondor. Em verde, personagens envolvidos na questão do regente de Gondor, que se recusava a sair do trono e tomou Pippin como um servo. Em laranja, o núcleo do anel, incluindo Frodo, Sam, Gollum e o vilão Sauron.

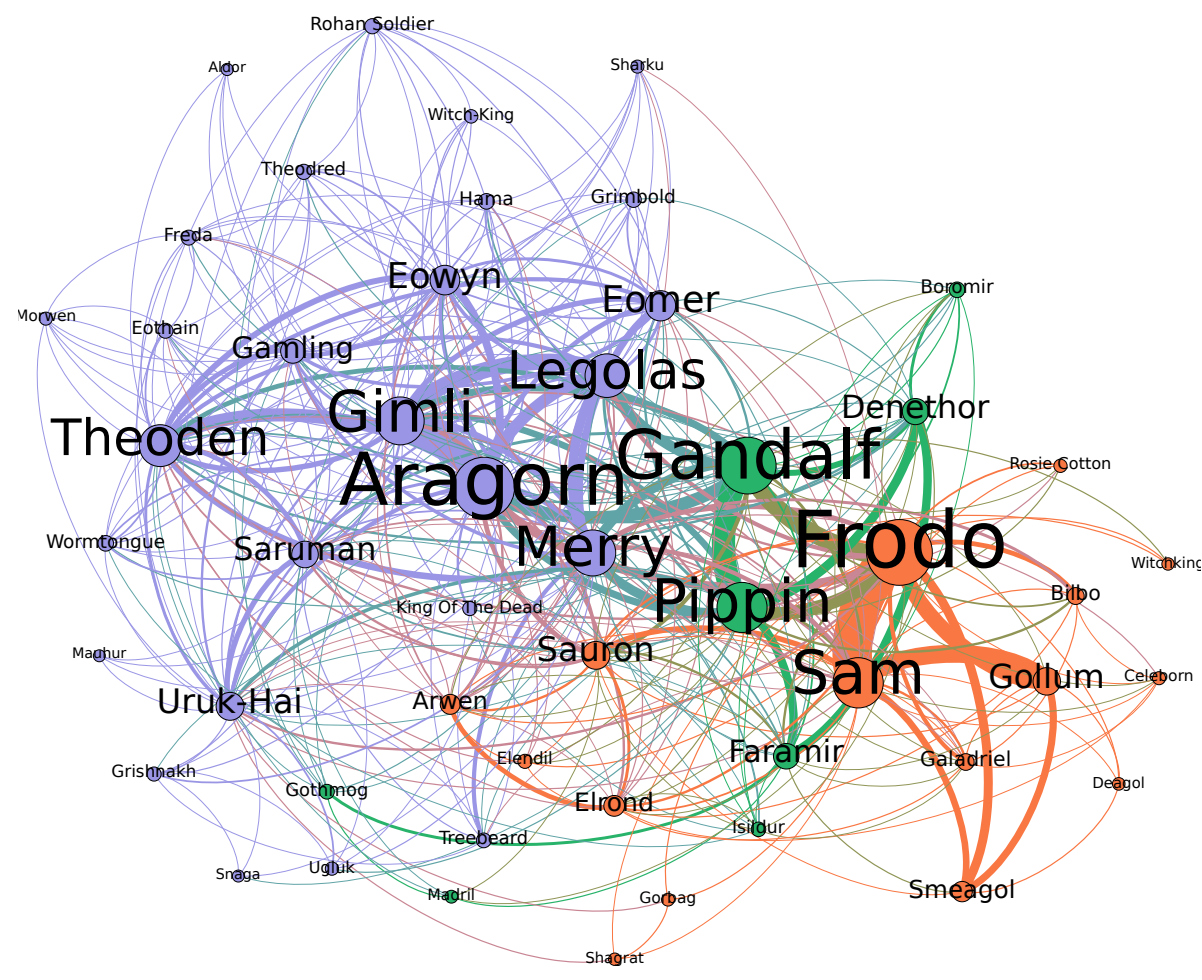

Figura 5. Rede de personagens da trilogia completa de 0 Senhor dos Aneis classificados de acordo com a centralidade de grau ponderado.

A classificação geral dos personagens na trilogia é exibida na Tabela 4. Os resultados indicam que os personagens Frodo, Aragorn e Gandalf são os protagonistas da história. Sam teve a maior pontuação em intermediação, devido à seu papel de ponte, conectando personagens de diferentes núcleos. Ele também obteve boas pontuações 
nas outras duas métricas, podendo ser considerado também um dos protagonistas. Esta classificação é condizente com a história dos três filmes: Frodo e Sam, os responsáveis pela destruição do anel; Aragorn, o rei; e Gandalf, o mago que possui papel de líder. É interessante observar que o fato de Sam ter se destacado é uma informação relevante e não esperada, pois durante grande parte das cenas, apesar de Sam estar presente, o foco é sempre Frodo. No entanto, as medidas indicam que o personagem também possui protagonismo na história.

Tabela 4. Classificação de personagens segundo as medidas de centralidade aplicadas à rede social da trilogia 0 Senhor dos Anéis.

\begin{tabular}{|l|l|}
\hline Personagens & $\begin{array}{l}\text { Grau } \\
\text { ponderado }\end{array}$ \\
\hline Frodo & 254.0 \\
\hline Aragorn & 232.0 \\
\hline Gandalf & 212.0 \\
\hline Sam & 182.0 \\
\hline Pippin & 181.0 \\
\hline Gimli & 171.0 \\
\hline Merry & 162.0 \\
\hline Legolas & 151.0 \\
\hline Theoden & 143.0 \\
\hline Eomer & 89.0 \\
\hline
\end{tabular}

\begin{tabular}{|l|l|}
\hline Personagens & Betweenness \\
\hline Sam & 0.08574 \\
\hline Uruk-Hai & 0.07858 \\
\hline Frodo & 0.07234 \\
\hline Aragorn & 0.07179 \\
\hline Merry & 0.06798 \\
\hline Gandalf & 0.06624 \\
\hline Theoden & 0.0455 \\
\hline Gimli & 0.04192 \\
\hline Saruman & 0.03919 \\
\hline Pippin & 0.03681 \\
\hline
\end{tabular}

\begin{tabular}{|l|l|}
\hline Personagens & PageRank \\
\hline Frodo & 0.07530 \\
\hline Aragorn & 0.07099 \\
\hline Gandalf & 0.06271 \\
\hline Sam & 0.05479 \\
\hline Pippin & 0.05434 \\
\hline Gimli & 0.05223 \\
\hline Merry & 0.05172 \\
\hline Theoden & 0.04613 \\
\hline Legolas & 0.04567 \\
\hline Uruk-Hai & 0.02996 \\
\hline
\end{tabular}

\section{Considerações finais}

Neste trabalho, introduzimos uma proposta para construção das redes sociais de personagens de um filme, e aplicamos especificamente nos três filmes da franquia O Senhor dos Anéis. A construção foi feita utilizando o script dos filmes, e considerando a participação de personagens que aparecem ou foram citados nas cenas com o auxílio da lista de personagens dos mesmos.

Após a construção das redes sociais, foi feita análise através de três medidas de centralidade: grau ponderado, intermediação e PageRank. Cada medida classifica os personagens que considera mais importantes de acordo com um critério específico. $\mathrm{O}$ objetivo foi verificar, segundo as métricas, quem são os protagonistas do filme e se a rede social obtida, bem como as comunidades identificadas e os personagens mais influentes são condizentes com o enredo do filme.

A classificação obtida das três medidas de centralidade aponta os personagens mais centrais na rede social de cada filme. Em A Sociedade do Anel, os protagonistas são Frodo, Sam e Gandalf. Em As Duas Torres, os destaques são Aragorn, Theoden e Sam. Já em $O$ Retorno do Rei, Frodo, Aragorn e Sam são os personagens mais influentes. Ao analisar os três filmes em conjunto, Frodo, Sam, Aragorn e Gandalf são os protagonistas apontados pelas medidas de centralidade.

Os resultados obtidos são condizentes com a história de cada filme, inclusive quando analisada a trilogia como um todo. As comunidades detectadas refletem também parte do roteiro, destacando núcleos que estão presentes na história. Desta forma, acreditamos que a abordagem escolhida é adequada para a modelagem da rede de personagens e pode ser aplicada a outros filmes. Como trabalhos futuros, pretendemos aplicar a técnica a filmes de outros gêneros e verificar se os vértices centrais de acordo com as medidas 
de centralidade são de fato os protagonistas, e se há alguma relação entre as redes sociais construídas a partir de filmes do mesmo gênero.

\section{Referências}

Agarwal, A., Balasubramanian, S., Zheng, J., and Dash, S. (2014). Parsing screenplays for extracting social networks from movies. EACL 2014, pages 50-58.

Bonato, A., D’Angelo, D. R., Elenberg, E. R., Gleich, D. F., and Hou, Y. (2016). Mining and modeling character networks. Algorithms and Models for the Web Graph, pages $100-114$.

Gephi (2017). Open graph viz platform. Disponível em: https://gephi .org/. Acessado em: 02/2017.

IMDb (2017). Internet movie database (imdb). Disponível em: http : / / www . imdb . com/. Acessado em: 02/2017.

IMSDb (2017). Internet movie script database (imsdb). Disponível em: http: / / www . imsdb.com/. Acessado em: 02/2017.

Kaminski, J., Schober, M., Albaladejo, R., Zastupailo, O., and Hidalgo, C. (2012). Moviegalaxies - social networks in movies.

Park, S.-B., Oh, K.-J., and Jo, G.-S. (2012). Social network analysis in a movie using character-net. Multimedia Tools and Applications, 59(2):601-627.

Perra, N. and Fortunato, S. (2008). Spectral centrality measures in complex networks. Physical Review E, 78(3):036107.

Shen, H.-W. (2013). Community structure of complex networks. Springer Science \& Business Media.

Spadon, G., Scabora, L. C., Araujo, M. V. S., Oliveira, P. H., Machado, B. B., de Sousa, E. P. M., Jr., C. T., and Jr., J. F. R. (2016). Complex network tools to understand the behavior of criminality in urban areas. CoRR, abs/1612.06115.

Weng, C.-Y., Chu, W.-T., and Wu, J.-L. (2007). Movie analysis based on roles' social network. In Multimedia and Expo, 2007 IEEE International Conference on, pages 1403-1406. IEEE. 